\title{
Ganetespib synergizes with cyclophosphamide to improve survival of mice with autochthonous tumors in a mutant p53-dependent manner
}

\author{
Evguenia M Alexandrova ${ }^{1}$, Sulan $\mathrm{Xu}^{1}$ and Ute M Mol${ }^{*, 1}$
}

The DNA-alkylating cytotoxic agent cyclophosphamide (CTX) is commonly used in the clinic to treat hematological malignancies like lymphomas and leukemias as well as solid tumors, but shows dose-dependent potentially life-threatening toxicities and can induce secondary malignancies. Thus, the clinical utility of CTX would be improved if a companion drug could be identified that allows lowering the CTX dose, while maintaining or even increasing its antineoplastic therapeutic efficacy. In mouse models, highdose CTX (300 mg/kg) is effective in treating T-lymphomas, while low dose (defined here as $100 \mathrm{mg} / \mathrm{kg}$ ) is ineffective. We previously showed that the HSP90 inhibitor ganetespib potently suppresses T-lymphoma initiation and progression and extends overall survival (OS) in hotspot knockin mice expressing the p53 gain-of-function mutants R175H and R248Q (mutp53) by 30-59\%. Here we asked whether ganetespib could potentiate the effect of low-dose CTX $(100 \mathrm{mg} / \mathrm{kg})$ in the autochthonous T-lymphoma-bearing mutp53 R248Q mouse model. Indeed, combinatorial CTX/ganetespib synergistically suppresses growth of autochthonous T-lymphomas in R248Q (p53Q/-) but not p53-I- control mice by reducing mutp53 levels and triggering apoptosis. Combinatorial treatment extends progression-free (PFS) and OS in p53Q/ - mice significantly longer than in p53-I- mice. Specifically, PFS of $\mathrm{p} 53 \mathrm{Q} /$ - mice improves 8.9-fold over CTX alone versus 3.6 -fold in p53 - I - mice. Likewise, OS of R248Q/ mice improves 3.6 -fold, but worsens in $\mathrm{p} 53-/-$ mice $(0.85$-fold) over CTX alone. Moreover, half of the $\mathrm{p} 53 \mathrm{Q} /-\mathrm{mice}$ on combinatorial treatment lived over 60 days, and one animal reached 121 days. In contrast, p53Q/ - mice on single-drug treatment and p53 - I- mice on any treatment lived less than 24 days. In sum, ganetespib synergizes with a sub-effective dose of CTX in mutp53 T-lymphomas by suppressing tumor growth and extending survival. Our results provide a potential strategy to reduce the effective clinical dose of CTX in mutant p53-bearing malignancies and attenuate CTX toxicity.

Cell Death and Disease (2017) 8, e2683; doi:10.1038/cddis.2017.108; published online 16 March 2017

Cyclophosphamide (CTX) is one of the most widely used chemotherapeutics, effective in human lymphomas (e.g., aggressive non-Hodgkin's lymphomas and follicular lymphoma) and leukemias (e.g., small lymphocytic leukemia SLL and chronic lymphocytic leukemia CLL) as well as solid tumors (e.g., breast and ovarian cancer, bone and soft tissue sarcomas). ${ }^{1}$ Moreover, due to its immunosuppressive actions, CTX is also used in bone marrow transplantation conditioning and mobilization regimens and in autoimmune diseases. ${ }^{2-4}$ However, as DNA-alkylating agent CTX shows dosedependent potentially life-threatening toxicities. High doses of CTX induce bone marrow suppression, cardiotoxicity ${ }^{5,6}$ (which is dose-limiting), gonadal failure in males and females $^{7,8}$ and bladder toxicity (hemorrhagic cystitis and fibrosis), ${ }^{9}$ and CTX is associated with induction of secondary malignancies (bladder cancer, acute leukemia and skin cancer). ${ }^{1,10}$ Thus, the clinical utility of CTX would be improved if a companion drug could be identified that allows lowering its dose while maintaining or even increasing its antineoplastic efficacy.

In mouse cancer models that express missense mutant p53, we and others previously showed that highly stabilized mutp53 gain-of-function proteins confer oncogenic addiction for tumor maintenance and metastasis. ${ }^{11-15}$ Conversely, mutp53 elimination significantly suppresses tumor growth and metastasis and markedly extends animal survival. ${ }^{16-18}$ Thus, in advanced autochthonous T-lymphomas genetic ablation of the human hotspot mutp53 R248Q allele triggers acute cancer cell apoptosis and suppresses tumor growth. ${ }^{15,16}$ Moreover, early allele ablation at 10 weeks of age (at the stage of organ-confined incipient disease) prevents clinical lymphoma development in most animals and extends survival by $37 \%{ }^{16}$

p53 is mutated in $~ 50 \%$ of all human malignancies and in $\sim 10 \%$ of hematological malignancies. ${ }^{19,20}$ The HSP9O chaperone machinery is a critical determinant for mutant p53 stability by protecting the conformationally aberrant mutp53 proteins from degradation through their E3 ubiquitin ligases Mdm2 and CHIP. ${ }^{21-24}$ Using the potent second-generation Hsp90 inhibitor ganetespib (Ganet, which showed a favorable safety profile in clinical trials), ${ }^{25}$ we found that Ganet triggers degradation of stabilized hotspot gain-of-function mutp53 $\mathrm{R} 172 \mathrm{H}^{11}$ and $\mathrm{R} 248 \mathrm{Q}^{15}$ in vitro and in vivo, accompanied by T-lymphoma apoptosis in both the allograft and autochthonous tumor setting. ${ }^{16}$ In clinical mouse trials, Ganet specifically extended survival of mutp53, but not p53null control mice, by

\footnotetext{
${ }^{1}$ Department of Pathology, Stony Brook University, Stony Brook, NY 11794, USA

*Corresponding author: UM Moll, Department of Pathology, Stony Brook University, 101 Nicolls Road, Stony Brook, NY 11794, USA. Tel: 631 444 2459; Fax: 631 444 2434; E-mail: Ute.Moll@ stonybrookmedicine.edu

Received 09.12.16; revised 14.2.17; accepted 15.2.17; Edited by G Melino
} 

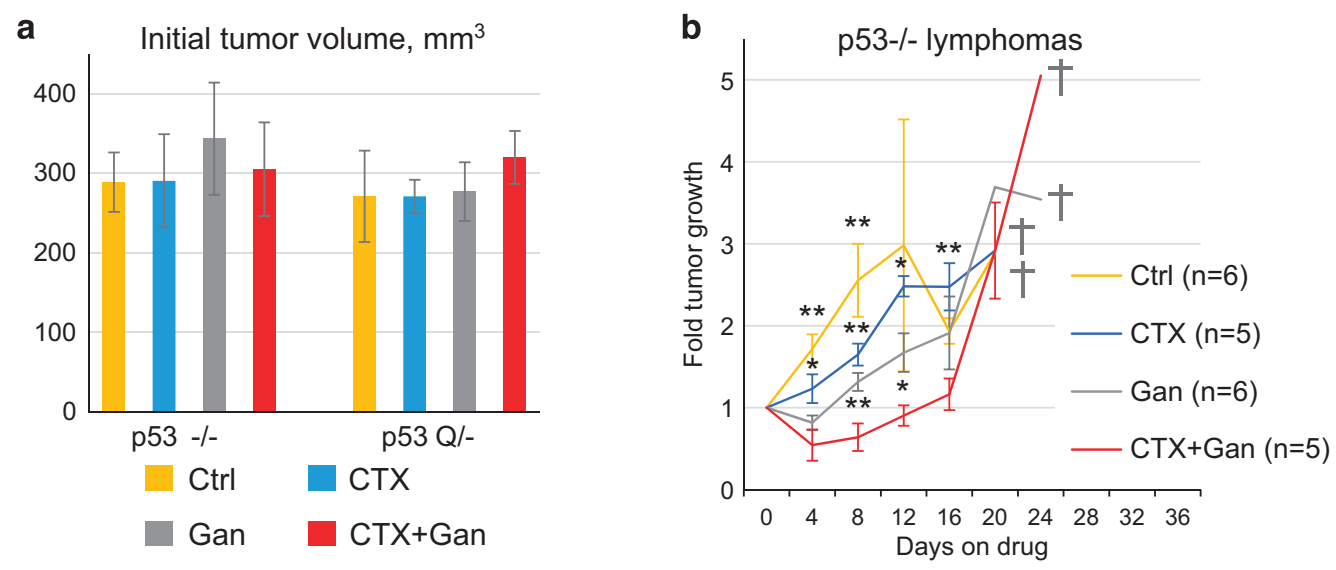

C

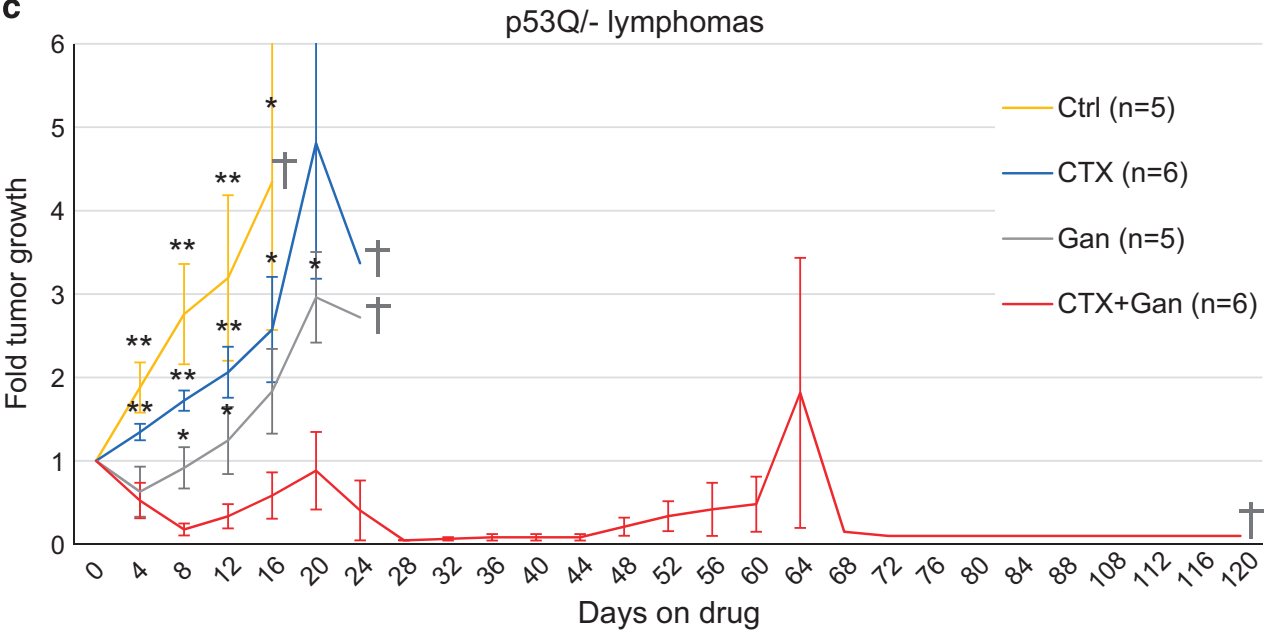

Figure 1 Treatment response of autochthonous T-lymphomas of p53 - / - and p53Q/ - mice, with or without single weekly doses of CTX alone (100 mg/kg), ganetespib alone (Gan, $50 \mathrm{mg} / \mathrm{kg}$ ) or both drugs in combination (100 mg/kg CTX+50 mg/kg Gan). (a) Tumor volume at treatment start; mean \pm S.E.M. (b and c) Tumor growth over time normalized to initial tumor size in $\mathrm{p} 53-/-$ (b) and $\mathrm{p} 53 \mathrm{Q} / \mathrm{-}$ - (c) animals. Mean \pm S.E.M.; $n$, number of mice; gray crosses, day when last animal in the group was killed; ${ }^{\star} P<0.05,{ }^{*} P<0.01$ relative to $C T X+G a n$

$48 \%$ in the $\mathrm{R} 175 \mathrm{H}$ cohorts and $59 \%$ in the $\mathrm{R} 248 \mathrm{Q}$ cohorts. ${ }^{16}$ CTX given at high dose $(300 \mathrm{mg} / \mathrm{kg})$ was previously shown to be effective in inhibiting tumor growth of T-lymphomas in p53null mice. ${ }^{26,27}$ Here we used the humanized (exons 4-9) mutp53 R248Q mouse model, which also primarily develops T-lymphoma, and report that Ganet synergizes with a low subeffective dose of CTX to effectively suppress tumor growth and extend animal survival in vivo. This data might support a general strategy to reduce the effective dose of CTX without compromising efficacy in anticancer therapy of mutp53expressing tumors.

\section{Results}

CTX given at high dose $(300 \mathrm{mg} / \mathrm{kg}$ ) was previously shown to be effective in inhibiting tumor growth of T-lymphomas in p53-/- mice. ${ }^{26,27}$ To test the effect of combinatorial treatment of low-dose CTX plus Ganet on the growth of autochthonous T-lymphomas in $\mathrm{p} 53 \mathrm{Q} / \mathrm{-}$ versus control p53-/ - mice and visualize tumor growth over time, we employed high-resolution ultrasound imaging. ${ }^{16}$ Thymus size and shape in p53Q/ - and p53 - / - control mice (about 40 per genotype) was examined starting at 8 weeks of age when the first T-lymphomas are known to arise. ${ }^{15,16}$ When individual tumors had reached $\sim 300 \mathrm{~mm}^{3}$ in volume (Figure 1a), each animal was randomly assigned to one of four treatment arms: (1) control (no treatment); (2) low-dose CTX alone $(100 \mathrm{mg} / \mathrm{kg}$ i.p.); (3) low-dose Ganet alone (50 mg/kg i.v.) and (4) combined CTX (100 mg/kg i.p.) and Ganet $(50 \mathrm{mg} / \mathrm{kg}$ i.v.). Mice were treated with the assigned drugs once a week, and lymphoma growth was monitored by sonography approximately every 4 days (Figures $1 \mathrm{~b}$ and $c$ and Figure 2). In agreement with our previous study, ${ }^{16}$ Ganet alone suppressed

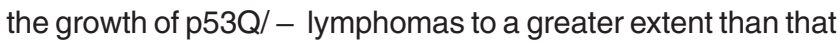
of p53-/ - lymphomas (Supplementary Figures 1a and b, gray versus yellow lines; Figures $1 b$ and $c$ ), although this did not reach statistical significance (data not shown). Although with Ganet alone both p53-/ - and p53Q/- Iymphomas resumed their growth after a short regression/stagnation period (Figures $1 b$ and $c$ and Figures $2 a$ and $b$ ), this time span was longer for $\mathrm{p} 53 \mathrm{Q} /$ - versus $\mathrm{p} 53$ - / - lymphomas ( 2 weeks versus 1 week, compare individual tumor growth in 
a
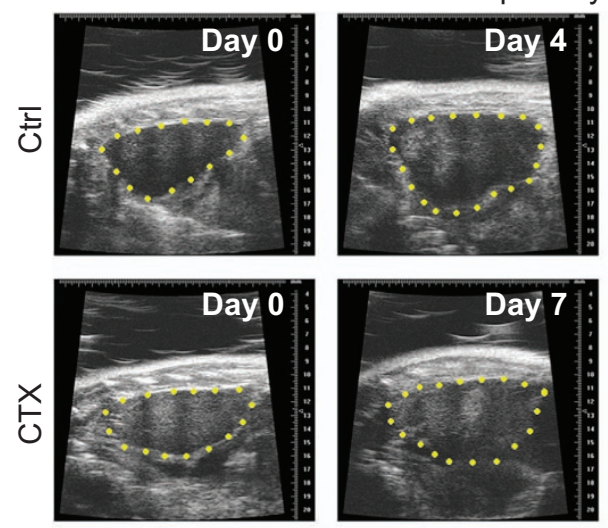

b
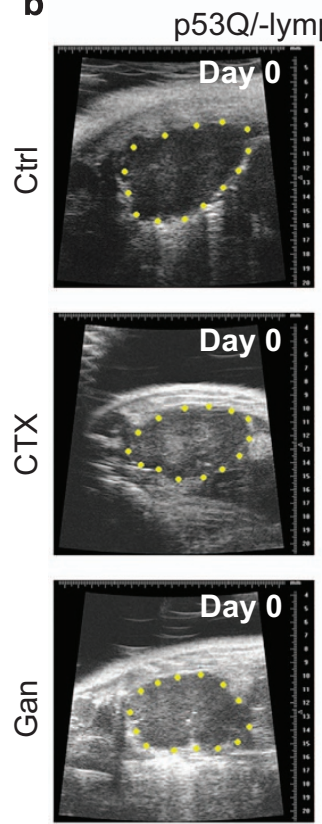
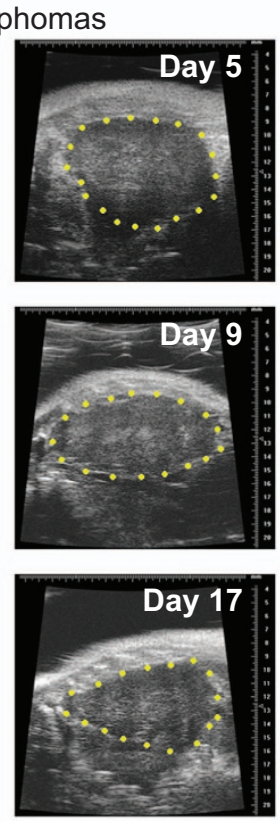

p53-/-lymphomas
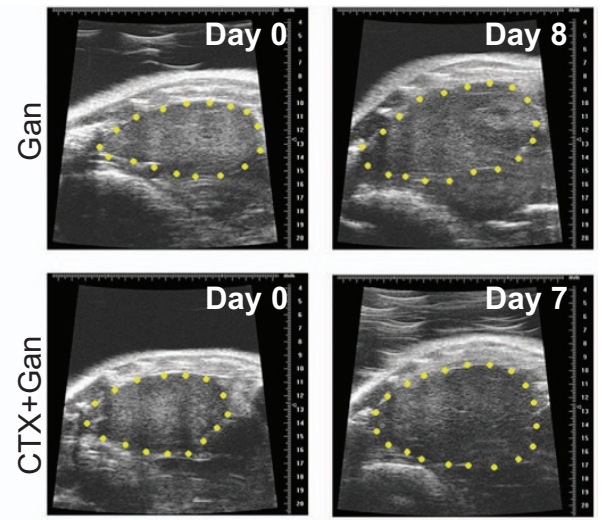

C
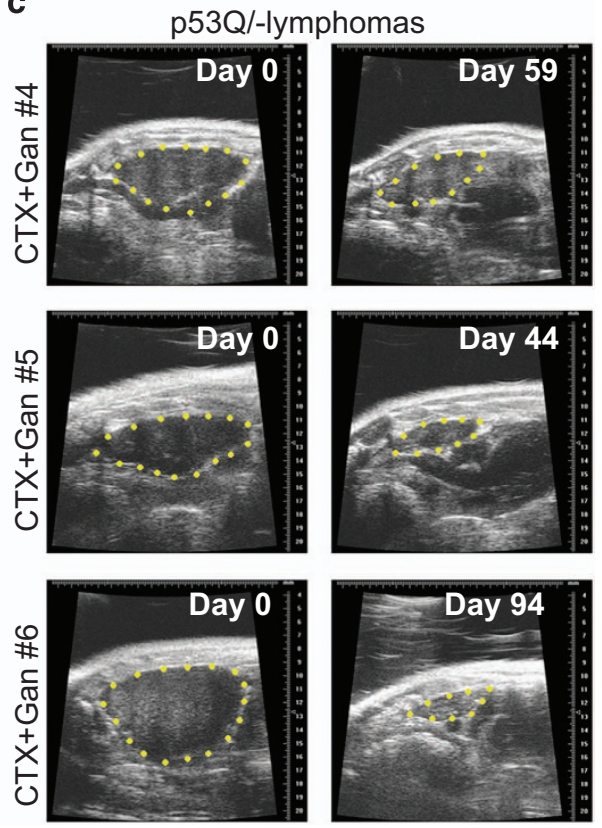

Figure 2 Representative high-resolution ultrasound images of autochthonous T-lymphomas of $\mathrm{p} 53-1-$ and $\mathrm{p} 53 \mathrm{Q} / \mathrm{-}$ mice, with or without weekly treatment with CTX alone (100 mg/kg), ganetespib alone (Gan, $50 \mathrm{mg} / \mathrm{kg}$ ) or both drugs in combination (100 mg/kg CTX+50 mg/kg Gan). (a) p53 - / - lymphomas, (b) p53Q/ - lymphomas, (c) p53Q/ lymphomas treated as indicated. Animal \# refer to mice in Table 1

Supplementary Figures $1 \mathrm{a}$ and $\mathrm{b}$ ), further confirming the previously observed mutp53-specific activity of Ganet. ${ }^{16}$ In contrast, due to the low-dose chosen, CTX alone suppressed tumor growth only mildly, and there was no difference in efficacy between $\mathrm{p} 53-/-$ and $\mathrm{p} 53 \mathrm{Q} / \mathrm{-}$ lymphomas (Figure 1b, blue lines; Supplementary Figures 1c and d, blue lines; Figures $2 a$ and $b$ ).

Strikingly however, when CTX and Ganet were combined at their respective single doses, the growth of $\mathrm{p53Q}$ - Iymphomas was dramatically suppressed. Moreover, this combinatorial efficacy occurred preferentially in mutp53 tumors compared to p53-/ - tumors (Figures $1 \mathrm{~b}$ and c, red lines; Supplementary Figures 1c and d, red lines; Figure 2c). Growth suppression of $\mathrm{p53Q} /$ - tumors was accompanied by degradation of stabilized mutp53 and induction of apoptosis as indicated by hematoxylin and eosin and cleaved caspase- 3 staining (Figure 3 and Supplementary Figure 2). Furthermore, while all untreated and single-drug-treated mice of both genotypes lived only up to a maximum of 24 days (Figures 1b and c; gray crosses indicate when the last mouse of the respective cohort died), half of the $\mathrm{p53Q} / \mathrm{-}$ animals (three out of six) treated with CTX+Ganet - but none of the respective p53-/- animals - lived for more than 60 days after treatment start (Figure 1c, red lines; Supplementary Figure 1d, red lines; Table 1). One p53Q/ - animal survived to 65 days with a small T-lymphoma but died of unknown cause (Table 1, mouse \#5). Notably, the longest p53Q/ - animal on CTX+Ganet (mouse \# 6, Figures $2 \mathrm{c}$ and $3 \mathrm{~d}$ ) lived for 121 days with a near-complete cure of its T-lymphoma at autopsy. Pathology analysis of the mediastinum of this mouse by hematoxylin and eosin staining revealed only normal thymic tissue, with rare single mutp53-positive tumor cells sprinkled 
a

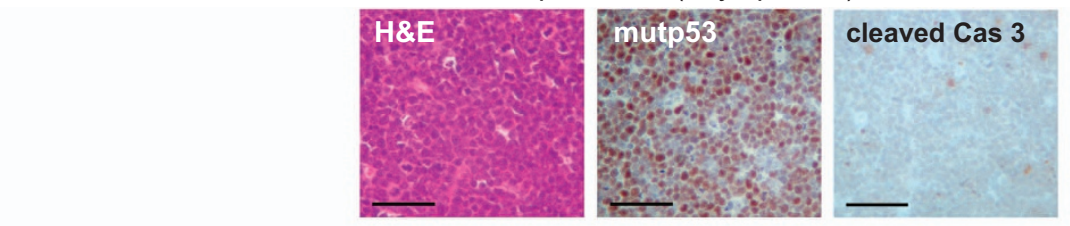

p53Q/-ctrl (T-lymphoma)

b

p53Q/-CTX+Gan, mouse \#1 (T-lymphoma)
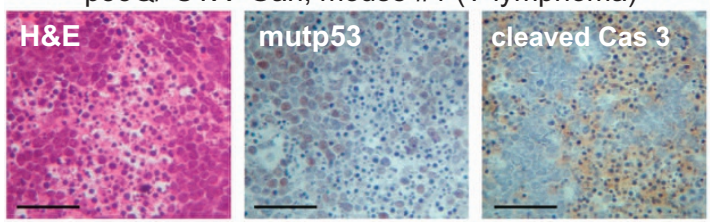

C

p53Q/-CTX+Gan, mouse \#3 (T-lymphoma)
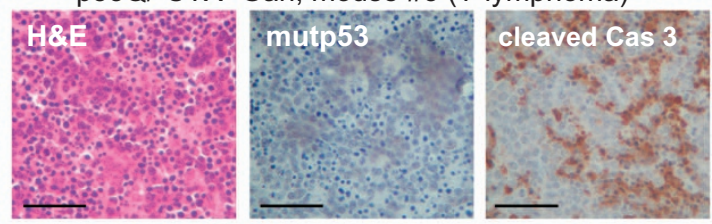

d

p53Q/-CTX+Gan, mouse \#6 (thymus)
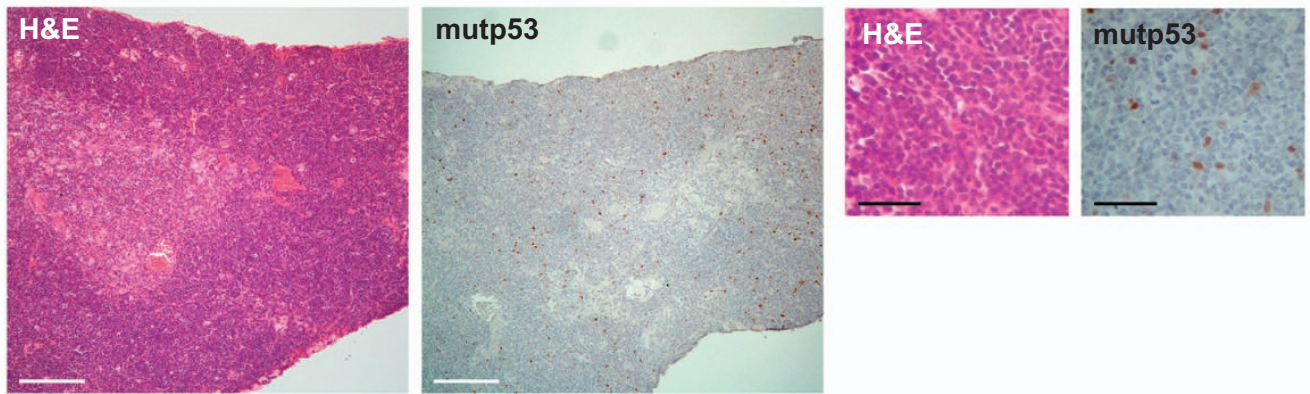

Figure 3 Representative T-lymphomas of $\mathrm{p53Q}$ - mice treated with combinatorial CTX+Gan, taken at end point. mutp53 levels, apoptosis (cleaved caspase-3) and histopathology. (a) Untreated control tumor. Nearly $100 \%$ of tumor cells express highly stabilized mutp53. (b-d) Mice treated with combined CTX+Gan. (b and c) Combined treatment induced strong depletion of mutp53 protein in tumors accompanied by massive apoptosis. (d) With combined treatment, the initially large T-lymphoma (see Figure 2c) rapidly regressed within 7 days to a nearly undetectable thymic structure and remained like this until death. At autopsy, only normal thymus was detected, which contained rare isolated mutp53-positive cells. The animal had to be killed after 121 days due to a small mutp53-positive fibrosarcoma obstructing the anus (see Supplementary Figure 2a). Immunostaining with the indicated antibodies. H\&E, hematoxylin and eosin. Black scale bar, $200 \mu \mathrm{m}$; white scale bar, $800 \mu \mathrm{m}$

Table 1 Response of p53 R248Q/ - mice bearing autochthonous T-lymphomas to combinatorial treatment with CTX (100 mg/kg) and ganetespib (50 mg/kg)

\begin{tabular}{lccl}
\hline $\begin{array}{l}\text { Mice treated with } \\
\text { CTX+Ganet }\end{array}$ & $\begin{array}{c}\text { OS after treatment } \\
\text { start, in days }\end{array}$ & $\begin{array}{c}\text { T-lymphoma growth at end point, } \\
\text { relative to initial size }\end{array}$ & Cause of death \\
\hline Mouse \#1 & 19 & 1 -fold & Interstitial pneumonia \\
Mouse \#2 & 19 & 2.6 -fold & T-lymphoma \\
Mouse \#3 & 26 & 1.5 -fold & $\begin{array}{l}\text { Lung atelectasis, T-lymphoma } \\
\text { Mouse \#4 }\end{array}$ \\
Mouse \#5 & 62 & 3.4 -fold & $\begin{array}{l}\text { T-lymphoma } \\
\text { Cause of death unknown. 11 days prior to death thymus } \\
\text { still in regression (0.3-fold of original tumor size) }\end{array}$ \\
Mouse \#6 & 65 & 0.1 -fold & Anal obstruction due to fibrosarcoma \\
\hline
\end{tabular}

${ }^{a}$ Censored in PFS and OS curves (Figures $4 b$ and $d$ and Figures $5 b$ and $d$ ) since death was not due to T-lymphoma

through the dense lymphoid tissue (Figure 3d). This animal had to be killed due to a small mutp53-positive fibrosarcoma that caused anal obstruction (Supplementary Figure $3 a$ and Table 1). Taken together, with the notable exception of the p53Q/ - cohort on combinatorial CTX+Ganet, pathological and immunohistochemical analyses showed that all other animals died from large recurrent T-lymphoma. These untreated control (Figure 3a) and single-drug-treated p53Q/ lymphomas (data not shown) exhibited highly stabilized mutp53. In contrast, only two out of six p53Q/ - animals on combinatorial CTX+Ganet died from large T-lymphomas (mice \#2 and \#4, Table 1 and Supplementary Figure 3b). Two additional animals (mouse \#1 and \#3) died from interstitial pneumonia and lung atelectasis, respectively (Supplementary Figures $3 \mathrm{c}$ and $\mathrm{d}$ ), and at autopsy had only small residual T-lymphomas with significantly reduced mutp53 levels and upregulated apoptosis (Figures $3 \mathrm{~b}$ and $\mathrm{c}$ and Table 1). Taken together, these data indicate that the combinatorial CTX +Ganet treatment is much more effective in suppressing growth of mutp53 compared to p53null T-lymphomas by destabilizing mutp53 protein levels and triggering apoptosis. Moreover, in mutp53Q/ - mice combinatorial treatment is also much more effective than CTX or Ganet alone.

Analysis of progression-free (PFS) (Figure 4) and overall survival (OS) (Figure 5) demonstrated a superior potency of Ganet in $\mathrm{p} 53 \mathrm{Q} /$ - versus p53-/ - animals (Figures $4 \mathrm{a}-\mathrm{c}$, 
a

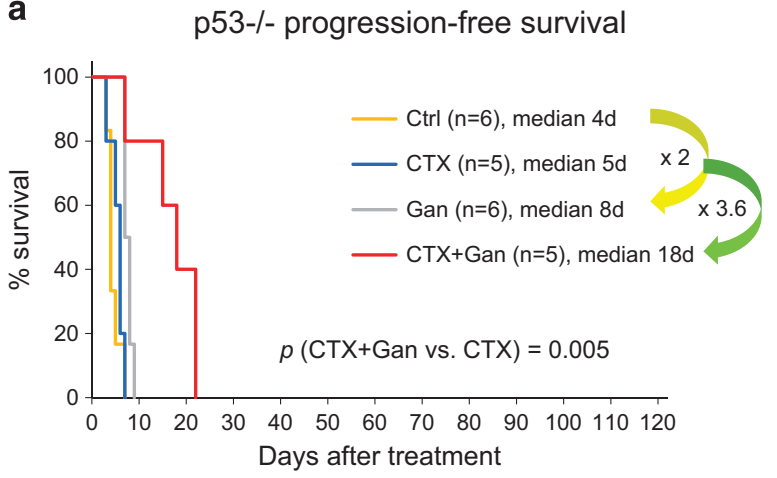

b

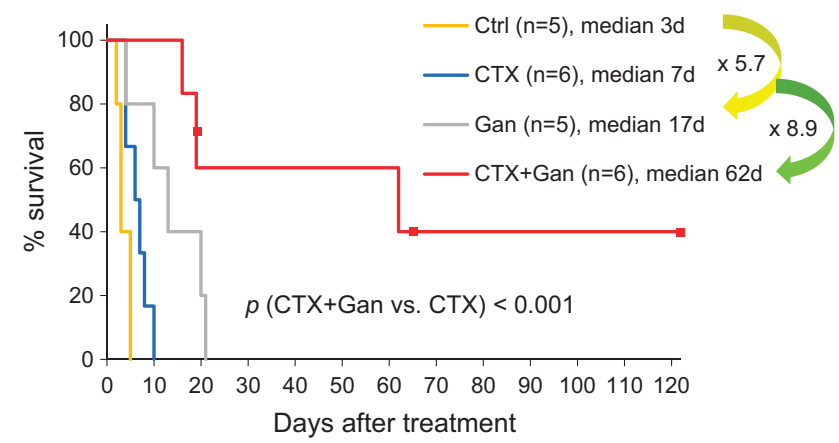

c

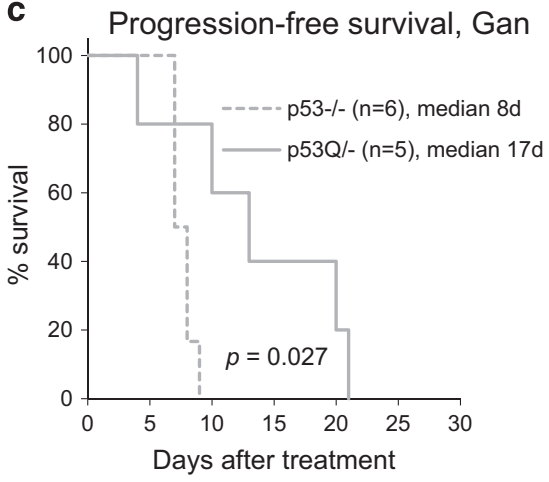

d Progression-free survival, CTX+Gan

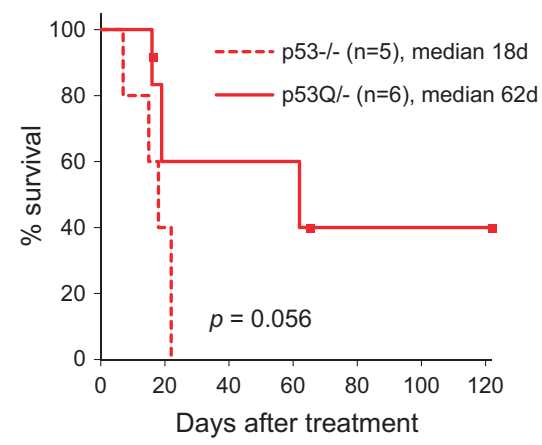

Figure 4 PFS of $p 53-/$ - and p53Q/ - animals with T-lymphoma (initial tumor size about $300 \mathrm{~mm}^{3}$, see Figure 1), with or without weekly treatment with CTX alone (100 mg/kg), ganetespib alone (Gan, $50 \mathrm{mg} / \mathrm{kg}$ ) or both drugs in combination (100 mg/kg CTX+50 mg/kg Gan). (a) p53 - / - animals. (b) p53Q/ - animals. (c and d) Paired $\mathrm{p53Q}$ - - versus p53 - / - analysis of PFS of Gan-treated mice (c) and CTX+Gan-treated mice (d) of the indicated genotypes. Kaplan-Meier analysis; $n$, number of mice; $p$, log rank statistics. Dots represent censored animals because they died of reasons other than T-lymphoma

yellow arrows and Figure 5c), confirming our previous findings of a relative mutp53 specificity of Ganet treatment. ${ }^{16}$ In contrast, CTX had a similar - albeit none or minimal - efficacy in both genotypes (Supplementary Figure 4). Most importantly, however, combinatorial CTX+Ganet prolonged PFS and OS significantly longer in $\mathrm{p} 53 \mathrm{Q} / \mathrm{-}$ than in p53-/animals. Compared to CTX alone, CTX+Ganet extended PFS and OS of p53Q/ - animals 8.9-fold (from 7 to 62 days) and 3.6-fold (from 17 to 62 days), respectively (Figures $4 \mathrm{~b}$ and $5 \mathrm{~b}$ ). In contrast, CTX+Ganet extended PFS of p53-/ - animals only 3.6-fold (from 5 to 18 days) relative to CTX alone, and failed to extend their OS (in fact, OS decreased from 26 to 22 days, 0.85 -fold) (Figures $4 \mathrm{a}$ and $5 \mathrm{a}$ ). Thus, upon combined treatment median PFS was 3.4 times longer (62 versus 18 days, $P=0.056$, Figure $4 d$ ) and median OS was 2.8 times longer (62 versus 22 days, $P=0.028$, Figure $5 d$ ) in $p 53 Q /-$ versus $\mathrm{p} 53$ - / - animals. All together, these data demonstrate strong cooperation between low-dose CTX and Ganet in extending PFS and OS specifically in mutp53 tumors in vivo.

\section{Discussion}

Here the gain-of-function hotspot mutant p53 R248Q mouse model, which primarily develops T-lymphomas, provided proof-of-principal that the Hsp90 inhibitor ganetespib potentiates the antineoplastic efficacy of CTX by suppressing autochthonous tumor growth and prolonging PFS and OS. These effects were significantly stronger in mutp53Q/ - than in p53null mice, suggesting that the combinatorial treatment might specifically benefit patients with mutp53-bearing malignancies. One obvious explanation is that Ganet alone prolongs the survival of mutp53 - but not p53null - animals by destabilizing mutp53 and triggering its proteasomal degradation. ${ }^{16}$ Indeed, with Ganet-only treatment, we already saw stronger effects in mutp53 compared to p53null animals, indicated by a longer period of tumor stagnation from 1 week to 2 weeks (compare individual tumor growth in Supplementary Figures 1a and b, gray lines) and by significantly extended PFS from 2- to 5.7-fold ( $P=0.027$, Figures $4 a-c)$. There is also a trend for improved OS in mutp53 versus p53null mice $(P=0.063$, Figure $5 \mathrm{c})$. A possible explanation why the differential effect of Ganet on mutp53 versus p53null animals was not quite as strong in this study as it was in our previous study $^{16}$ is that previous treatment was started at 8 weeks of age at the stage of incipient disease with normal-sized thymus, while here treatment was started at the stage of advanced tumors ( $300 \mathrm{~mm}^{3}$ in volume) at a median of 20 weeks (data not shown), shortening drug exposure. Moreover, in contrast to the previous study, the mutp53 versus p53null cohorts used here were not littermates.

Another possible explanation why combinatorial CTX +Ganet preferentially affects mutp53Q/ - versus p53null tumors is that mutp53Q/ - cancer cells proliferate faster than p53null cells, since growth advantage over simple p53 absence is one of the phenotypes of mutp53 gain-offunction. ${ }^{15}$ This could result in their higher sensitivity to the 
a

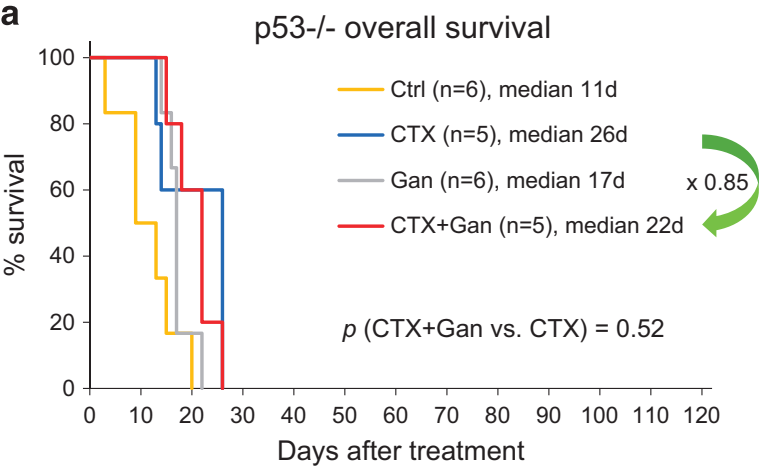

b

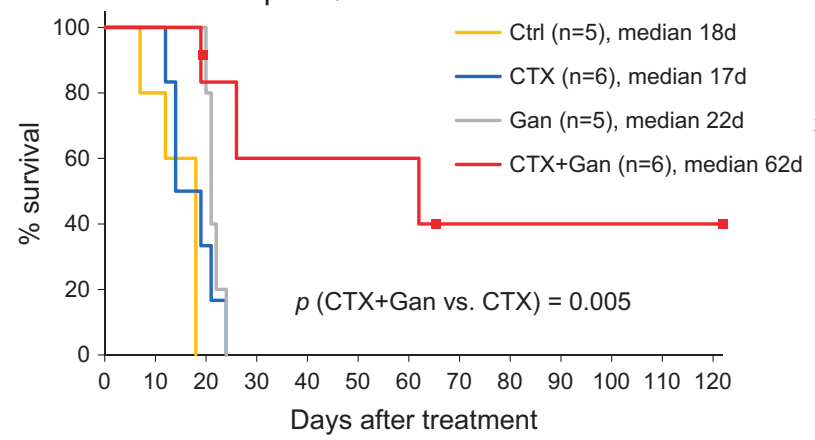

C

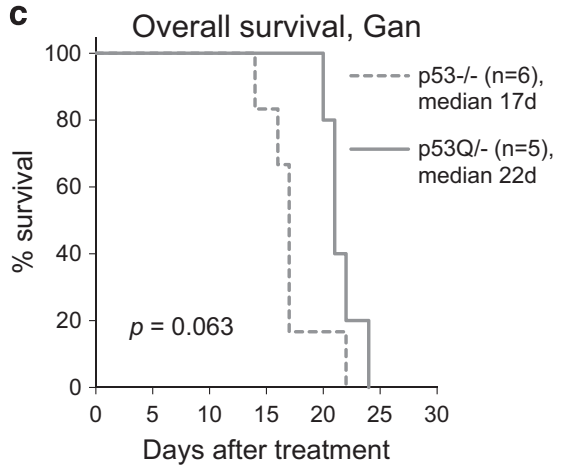

d

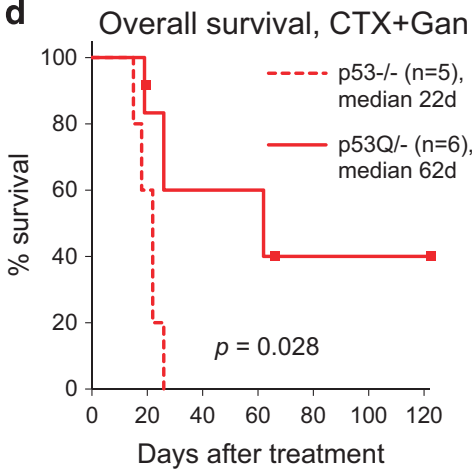

Figure 5 OS of the same p53 - / - and p53Q/ - animals with T-lymphoma as in Figure 4, with or without weekly treatment with CTX alone (100 mg/kg), ganetespib alone (Gan, $50 \mathrm{mg} / \mathrm{kg}$ ) or both drugs in combination (100 mg/kg CTX+50 mg/kg Gan). (a) p53 - / - animals. (b) p53Q/ - animals. (c and d) Paired p53Q/ - versus p53 - / analysis of OS of Gan-treated mice (c) and CTX+Gan-treated mice (d) of the indicated genotypes. Kaplan-Meier analysis; $n$, number of mice; $p$, log rank statistics. Dots represent censored animals

DNA-alkylating CTX. However, we did not see preferential sensitivity of mutp53Q/ - mice versus p53null mice to CTX alone (Supplementary Figure 4). It seems that in combination, CTX and Ganet potentiate each other's efficacy and specificity toward mutp53-bearing tumors. It remains to be established whether CTX and Ganet will also synergize to suppress autochthonous tumors expressing different stabilized p53 missense mutants besides the hotspot R248Q described here. A potent and fast format for predicting efficacy against a whole spectrum of different p53 mutants is subcutaneous T-lymphoma allografts. Based on this, the likelihood for broader efficacy beyond $\mathrm{R} 248 \mathrm{Q}$ is high, considering that $\mathrm{CTX}+\mathrm{Ganet}$ synergism was also seen toward gain-of-function mutp53 $\mathrm{R} 175 \mathrm{H}^{16}$

Interestingly, treatment of either mutp53 or p53null animals with Ganet alone invariably results in tumor resistance and relapse (Figures 1a and b and Supplementary Figures 1a and b, gray lines). In human colorectal carcinoma cells, we previously described a Ganet resistance mechanism via elevated expression of UGT1A, a glucoronidating enzyme that causes excretion of Ganet into the extracellular space, eliminating its ability to inhibit intracellular Hsp90. ${ }^{28}$ However, this mechanism does not seem to be relevant in mutp53driven T-lymphomas. ${ }^{16}$ Interestingly, however, in the combinatorial $\mathrm{CTX}+$ Ganet treatment arm, CTX completely prevented Ganet resistance in at least two out of six mutp53 animals (Table 1 and Supplementary Figure 1d, mice \#5 and \#6), further attesting to the mutp53-specific synthetic lethality of Ganet and CTX. While the mechanism of this synergistic effect and the generality of cytotoxic drugs preventing resistance to Ganet remain to be elucidated, our findings point toward the exciting possibility that the combination CTX+Ganet will facilitate both, to lower the effective CTX dose and side effects and to overcome resistance to Ganet.

\section{Materials and Methods}

Mice and high-resolution ultrasound imaging. Hotspot humanized mutp53 R248Q mice and p53-/- control mice were previously described. ${ }^{15}$ Parental p53 R248Q/+ and p53-/+ strains, both on mixed 129SvImJ/C57BI6J background, were intercrossed to obtain p53 R248Q/ - and p53-/ - mice. Animals were monitored weekly by ultrasound imaging, using the Visual Sonics Vevo 770 High-Resolution Imaging System. When tumors had reached $300 \mathrm{~mm}^{3}$ on average (ranging from 136 to $506 \mathrm{~mm}^{3}$ ), single weekly drug treatment was initiated as described below, followed by ultrasound imaging approximately every 4 days. Sagittal and transverse images were taken using the Vevo 770 V3.0.0 software. Tumor volume was calculated according to the formula for ellipsoid, $V=\pi / 6 \times$ height $\times$ width $\times$ length. Mice were killed at end point, defined as appearing moribund. Note that the T-lymphoma size at end point was different for different animals, but on average was similar between treatment groups (Supplementary Figure 2a), except for $\mathrm{p53Q}$ - mice treated with combined drugs, as explained in 'Results' section. This variability is due to the fact that the clinical signs of the disease that require killing (labored breathing eventually leading to suffocation) correlate not only with tumor size, but also with tumor shape and compression effects on the cardio-vascular and pulmonary system depending on the specific location in the chest cavity. These parameters are variable and can cause the moribund state even when T-lymphoma size is not that enlarged. All animals were treated humanely and according to the guidelines issued by the Institutional Animal Care and Use Committee at Stony Brook University.

CTX and ganetespib treatment. CTX (cyclophosphamide monohydrate, Sigma, C0768) was dissolved in phosphate-buffered saline and injected 
intraperitoneally at $100 \mathrm{mg} / \mathrm{kg}$ once a week. Ganetespib (STA-9090, Synta Pharmaceuticals, Lexington, MA, USA) was prepared as previously described ${ }^{16,29}$ and injected into the tail vein at $50 \mathrm{mg} / \mathrm{kg}$ once a week. For combinatorial treatment, animals were injected with $100 \mathrm{mg} / \mathrm{kg}$ CTX plus $50 \mathrm{mg} / \mathrm{kg}$ ganetespib within $5 \mathrm{~min}$ of each other.

Immunohistochemistry and histology. For immunohistochemical analysis, freshly dissected tissues were formalin fixed, paraffin embedded and sectioned $(5 \mu \mathrm{m})$. Slides were deparaffinized and boiled in citrate buffer $(10 \mathrm{mM}, \mathrm{pH}$ $6.0,35 \mathrm{~min}$ ) for antigen retrieval, blocked in $10 \%$ goat serum and incubated with primary antibodies (mutp53, Santa Cruz FL393, Dallas, TX, USA, sc-6243, dilution 1:500; cleaved caspase-3, Cell Signaling (Danvers, MA, USA), 9661, dilution $1: 500)$ for $2 \mathrm{~h}$ at room temperature. After PBS washing, slides were incubated with biotinylated secondary antibody and HRP-streptavidin using the Histostain SP Broad Spectrum kit (Invitrogen, Carlsbad, CA, USA, 959943B), stained with DAB substrate with hematoxylin counterstain and coverslipped. Other sections were stained by hematoxylin and eosin.

Statistical analysis. Unpaired two-tailed Student's t-test was used to analyze tumor size, percent of apoptotic cells and percent of mutp53-positive cells. KaplanMeier analysis with log rank statistics was used to analyze PFS and OS. $P<0.05$ was considered statistically significant.

\section{Conflict of Interest}

The authors declare no conflict of interest.

Acknowledgements. This work was supported by NCI Grant R01CA176647, TRO Grant Walk for Beauty (Stony Brook Foundation) and Wilhelm Sander Stiftung Grant 2011.029.1 to UMM and NCI Grant K22CA190653-01A1 to EMA.

1. Emadi A, Jones RJ, Brodsky RA. Cyclophosphamide and cancer: golden anniversary". Nat Rev Clin Oncol 2009; 6: 638-647.

2. Santos GW. Busulfan and cyclophosphamide versus cyclophosphamide and total body irradiation for marrow transplantation in chronic myelogenous leukemia - a review. Leuk Lymphoma 1993; 11: 201-204.

3. Salinger DH, McCune JS, Ren AG, Shen DD, Slattery JT, Phillips B et al. Real-time dose adjustment of cyclophosphamide in a preparative regimen for hematopoietic cell transplant: a Bayesian pharmacokinetic approach. Clin Cancer Res 2006; 12: 4888-4898.

4. Teles KA, Medeiros-Souza P, Lima FA, Araújo BG, Lima RA. Cyclophosphamide administration routine in autoimmune rheumatic diseases: a review. Rev Bras Reumatol 2016 (in press).

5. Ayash LJ, Wright JE, Tretyakov O, Gonin R, Elias A, Wheeler C et al. Cyclophosphamide pharmacokinetics: correlation with cardiac toxicity and tumor response. J Clin Oncol 1992; 10: $995-1000$.

6. Murdych T, Weisdorf DJ. Serious cardiac complications during bone marrow transplantation at the University of Minnesota, 1977-1997. Bone Marrow Transplant 2001; 28: 283-287.

7. Cigni A, Faedda R, Atzeni MM, Pileri PV, Alagna S, Rovasio P et al. Hormonal strategies for fertility preservation in patients receiving cyclophosphamide to treat glomerulonephritis: a nonrandomized trial and review of the literature. Am J Kidney Dis 2008; 52: 887-896.

8. Dooley MA, Nair R. Therapy insight: preserving fertility in cyclophosphamide-treated patients with rheumatic disease. Nat Clin Pract Rheumatol 2008; 4: 250-257.

9. Matz EL, Hsieh MH. Review of advances in uroprotective agents for cyclophosphamide- and ifosfamide-induced hemorrhagic cystitis. Urology 2017; 100: 16-19.

10. Kempen JH, Gangaputra S, Daniel E, Levy-Clarke GA, Nussenblatt RB, Rosenbaum JT et al. Long-term risk of malignancy among patients treated with immunosuppressive agents for ocular inflammation: a critical assessment of the evidence. Am J Ophthalmol 2008; 146: 802-812.

11. Lang GA, Iwakuma T, Suh YA, Liu G, Rao VA, Parant JM et al. Gain of function of a p53 hot spot mutation in a mouse model of Li-Fraumeni syndrome. Cell 2004; 119: 861-872.
12. Olive KP, Tuveson DA, Ruhe ZC, Yin B, Willis NA, Bronson RT et al. Mutant p53 gain of function in two mouse models of Li-Fraumeni syndrome. Cell 2004; 119: 847-860.

13. Morton JP, Timpson P, Karim SA, Ridgway RA, Athineos D, Doyle B et al. Mutant p53 drives metastasis and overcomes growth arrest/senescence in pancreatic cancer. Proc Natl Acad Sci USA 2010; 107: 246-251.

14. Doyle B, Morton JP, Delaney DW, Ridgway RA, Wilkins JA, Sansom OJ. p53 mutation and loss have different effects on tumourigenesis in a novel mouse model of pleomorphic rhabdomyosarcoma. J Pathol 2010; 222: 129-137.

15. Hanel W, Marchenko N, Xu S, Yu SX, Weng W, Moll U. Two hot spot mutant p53 mouse models display differential gain of function in tumorigenesis. Cell Death Differ 2013; 20 : 898-909.

16. Alexandrova EM, Yallowitz AR, Li D, Xu S, Schulz R, Proia DA et al. Improving survival by exploiting tumour dependence on stabilized mutant p53 for treatment. Nature 2015; $\mathbf{5 2 3}$ 352-356.

17. Weissmueller S, Manchado E, Saborowski M, Morris JP 4th, Wagenblast E, Davis CA et al. Mutant p53 drives pancreatic cancer metastasis through cell-autonomous PDGF recepto beta signaling. Cell 2014; 157: 382-394.

18. Muller PA, Vousden KH. Mutant p53 in cancer: new functions and therapeutic opportunities. Cancer Cell 2014; 25: 304-317.

19. Brosh R, Rotter V. When mutants gain new powers: news from the mutant $\mathrm{p} 53$ field. Nat Rev Cancer 2009; 9: 701-713.

20. Peller S, Rotter V. TP53 in hematological cancer: low incidence of mutations with significant clinical relevance. Hum Mutat 2003; 21: 277-284.

21. Blagosklonny MV, Toretsky J, Bohen S, Neckers L. Mutant conformation of p53 translated in vitro or in vivo requires functional HSP90. Proc Natl Acad Sci USA 1996; 93 : 8379-8383.

22. Whitesell L, Sutphin PD, Pulcini EJ, Martinez JD, Cook PH. The physical association of multiple molecular chaperone proteins with mutant p53 is altered by geldanamycin, an hsp90-binding agent. Mol Cell Biol 1998; 18: 1517-1524.

23. Li D, Marchenko ND, Moll UM. SAHA shows preferential cytotoxicity in mutant p53 cancer cells by destabilizing mutant $\mathrm{p} 53$ through inhibition of the HDAC6-Hsp90 chaperone axis. Cell Death Differ 2011; 18: 1904-1913.

24. Li D, Marchenko ND, Schulz R, Fischer V, Velasco-Hernandez T, Talos F et al. Functional inactivation of endogenous MDM2 and CHIP by HSP90 causes aberrant stabilization of mutant p53 in human cancer cells. Mol Cancer Res 2011; 9: 577-588.

25. Proia DA, Bates RC. Ganetespib and HSP90: translating preclinical hypotheses into clinical promise. Cancer Res 2014; 74: 1294-1300.

26. Schmitt CA, McCurrach ME, de Stanchina E, Wallace-Brodeur RR, Lowe SW. INK4a/ARF mutations accelerate lymphomagenesis and promote chemoresistance by disabling p53. Genes Dev 1999; 13: 2670-2677.

27. Schmitt CA, Fridman JS, Yang M, Lee S, Baranov E, Hoffman RM et al. A senescence program controlled by $\mathrm{p} 53$ and p16INK4a contributes to the outcome of cancer therapy. Cell 2002; 109: 335-346.

28. Landmann $H$, Proia DA, He S, Ogawa LS, Kramer F, Beißbarth $T$ et al. UDP glucuronosyltransferase $1 \mathrm{~A}$ expression levels determine the response of colorectal cancer cells to the heat shock protein 90 inhibitor ganetespib. Cell Death Dis 2014; 5: e1411.

29. Proia DA, Foley KP, Korbut T, Sang J, Smith D, Bates RC et al. Multifaceted intervention by the Hsp90 inhibitor ganetespib (STA-9090) in cancer cells with activated JAK/STAT signaling. PLOS ONE 2011; 6: e18552.

(i) Cell Death and Disease is an open-access journal published by Nature Publishing Group. This work is licensed under a Creative Commons Attribution 4.0 International License. The images or other third party material in this article are included in the article's Creative Commons license, unless indicated otherwise in the credit line; if the material is not included under the Creative Commons license, users will need to obtain permission from the license holder to reproduce the material. To view a copy of this license, visit http://creativecommons.org/licenses/by/4.0/

(C) The Author(s) 2017 